\title{
Selectivity of amidrazones towards activated nitriles - synthesis of new pyrazoles and NMR investigation
}

\author{
Ashraf A. Aly, ${ }^{\text {a* }}$ Alan B. Brown, ${ }^{\text {b Mohamad Ramadan, }}{ }^{\text {c }}$ \\ Mohamed Abd El-Aziz, ${ }^{\text {S Stefan Bräse }}{ }^{\mathrm{e}}$ and Hazem M. Fathy ${ }^{\mathrm{d}}$ \\ ${ }^{a}$ Chemistry Department, Faculty of Science, Minia University, 61519 El-Minia, A. R. Egypt \\ ${ }^{b}$ Chemistry Department, Florida Institute of Technology, Melbourne, FL 32901, USA \\ ${ }^{c}$ Department of Organic Chemistry, Faculty of Pharmacy, Al-Azhar University, Assiut Branch, \\ Egypt \\ ${ }^{d}$ Medicinal Department, Faculty of Pharmacy, Minia University, 61519 El Minia, Egypt \\ ${ }^{e}$ Institute of Organic Chemistry, Karlsruhe Institute of Technology, Fritz-Haber-Weg 6, 76131 \\ Karlsruhe, Germany \\ E-mail: ashrafaly63@yahoo.com
}

DOI: https://doi.org/10.24820/ark.5550190.p009.877

\begin{abstract}
$N$-Arylbenzamidrazones reacted with 2-(ethoxymethylene)malononitriles in ethanol/DMF (20:1), catalyzed by triethylamine $\left(\mathrm{Et}_{3} \mathrm{~N}\right)$, to give $65-81 \%$ yields of the corresponding pyrazoles. The structures of the products were proved by IR, mass, and NMR spectra and elemental analyses. Two-dimensional NMR correlations were used to differentiate between possible structures. The reaction mechanism is also discussed.
\end{abstract}

Keywords: Amidrazones, 2-(ethoxymethylene)malononitriles, pyrazoles, NMR, conjugate addition

\section{Introduction}

Amidrazones are convenient building blocks for various heterocycles. ${ }^{1-5}$ Recently, we have reported that amidrazones reacted with 2,3-diphenylcyclopropenone via extrusion of ammonia to give 3-(aryl)-2,5,6-triphenylpyrimidin-4(3H)-ones. ${ }^{6}$

For a very long time, the usefulness and great therapeutic value of the pyrazole nucleus have been recognized and the activities of this nucleus have been evaluated. ${ }^{7}$ Phenazone (antipyrine), a pyrazolin-5-one, was one of the first synthetic drugs. ${ }^{8}$ Phenylbutazone, a pyrazolidinedione, is a very potent anti-inflammatory agent, but its use is now banned in some countries. ${ }^{9}$ Later on, many modifications of the pyrazole nucleus were attempted and several compounds have been 
synthesized for the treatment of different conditions like inflammation, pain, cancer, tuberculosis, and diseases caused by bacteria. Some pyrazoles have shown high anti-inflammatory activity compared to indomethacin as the standard drug. ${ }^{10}$ Tewari et al. ${ }^{11}$ have synthesized a series of pyrazoles and evaluated them in vivo for their anti-inflammatory activity in carrageenan-induced rat paw edema model using nimesulide as the standard drug. Molecular modeling studies showed that pyrazole analogs interact with the cyclooxygenase-2 (COX-2) active site by classical hydrogen bonding, $\pi-\pi$ interaction, and cation $-\pi$ interaction which increases the residence of the ligand in the active site, consequently augmenting the anti-inflammatory activity of the compounds. ${ }^{11}$ Anti-bacterial activity was also tested by Ragavan et al., ${ }^{12}$ who have synthesized a group of 1,5-diaryl pyrazoles by altering the active part (amide linkage) and tested the products for anti-bacterial activity against E. coli (American Type Culture Collection [ATTC] - 25922), $S$. aureus (ATTC-25923), Pseudomonas aeruginosa (ATTC-27853), and Klebsiella pneumonia. As anti-cancer agents, pyrazoles have potent anti-angiogenic activity, and inhibit the growth of human breast (MCF-7) and cervical (Hela) carcinoma cells in vitro. ${ }^{13}$ Also, it was reported that some of a series of 1-arylmethyl-3-aryl-1H-pyrazole-5-carbohydrazide derivatives inhibit the growth of A549 cells, and induce cell apoptosis. ${ }^{14}$ In addition, two series of pyrazoles were studied as inhibitors of epidermal growth factor receptor (EGFR) kinase; the most potent had an $\mathrm{IC}_{50}$ of 0.07 $\mu \mathrm{M}$, as compared to positive control erlotinib. ${ }^{15}$ As antidepressant and anticonvulsant agents, pyrazoles showed good activity as compared to imipramine at a dose level of $10 \mathrm{mg} / \mathrm{kg}$. ${ }^{16}$ Pyrazoles have also found use as bifunctional ligands for metal catalysis, ${ }^{17,18}$ as model systems to study excited-state intramolecular proton transfers, ${ }^{19}$ as artificial receptors, ${ }^{20,21}$ and as the backbone to numerous scorpionate ligands. ${ }^{22}$ For all these reasons, pyrazole synthesis continues to attract interest. Herein we describe the synthesis of new pyrazoles via the reaction of amidrazones with activated alkenes.

\section{Results and Discussion}

Amidrazones $^{23}$ 1a-g reacted with 2-(ethoxymethylene)malononitriles 2a-c in ethanol/DMF (20:1), in presence of triethylamine $\left(\mathrm{Et}_{3} \mathrm{~N}\right)$ to furnish the pyrazoles 3a-o in $65-81 \%$ yields (Scheme 1). We chose amidrazones 1a-g having aryl groups with either electron-donating or electron withdrawing substitutents on the benzene ring, in order to examine their effect on the reaction. Amidrazones bearing electron-donating groups in the phenyl group attached to $N^{3}$ such as $p$-Me (1b), 2,6-di-Me (1c) or 2-Me (1g), or moderately electron-withdrawing substituents such as $p$-Cl (1d) or $p-\operatorname{Br}(\mathbf{1 f})$, all underwent the reaction smoothly to give the respective pyrazoles $(\mathbf{3 b}-\mathbf{d}, \mathbf{f}-\mathbf{h}, \mathbf{j}-$ $\mathbf{m , o}$ ) in $70-81 \%$ yields. For amidrazones bearing strong electron-withdrawing substituents in $N^{3}$ Ar such as $p-\mathrm{NO}_{2}(\mathbf{1 e})$, the reactions proceeded slowly to give 65-67\% yields of the corresponding pyrazoles $(\mathbf{3 e}, \mathbf{i}, \mathbf{n})$.

Elemental analyses and IR, NMR, and mass spectra were in good agreement with the assigned product structures. Structures were elucidated with the aid of $1 \mathrm{D}{ }^{1} \mathrm{H}$ and ${ }^{13} \mathrm{C}$ NMR spectra, and 
${ }^{1} \mathrm{H}-{ }^{1} \mathrm{H}$ COSY and ${ }^{1} \mathrm{H}_{-}{ }^{13} \mathrm{C}$ and ${ }^{1} \mathrm{H}-{ }^{15} \mathrm{~N}$ HSQC and HMBC correlations. Figure 1 shows the positional designations used throughout the series.

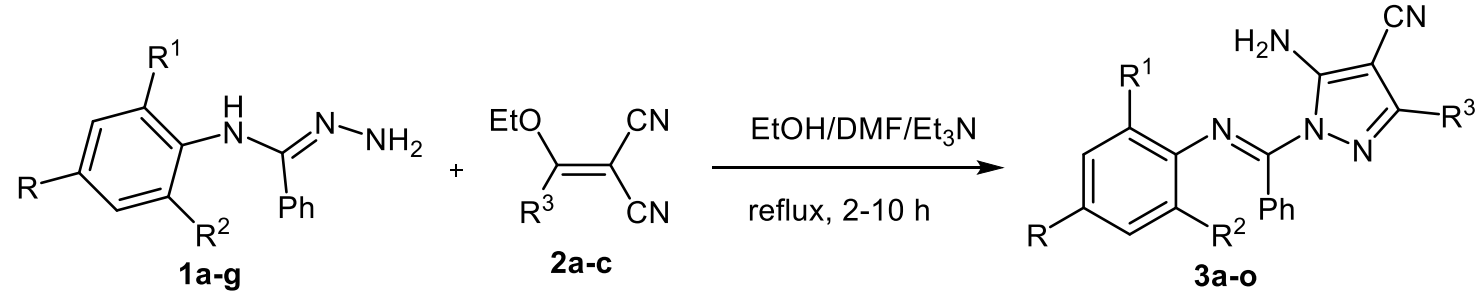

\begin{tabular}{|c|c|}
\hline $\begin{array}{l}\text { 1a: } R=R^{1}=R^{2}=H \\
\text { 1b: } R=C H_{3}, R^{1}=R^{2}=H \\
\text { 1c: } R=H, R^{1}=R^{2}=C_{3} \\
\text { 1d: } R=C l, R^{1}=R^{2}=H \\
\text { 1e: } R=N_{2}, R^{1}=R^{2}=H \\
\text { 1f: } R=B r, R^{1}=R^{2}=H \\
\text { 1g: } R=R^{2}=H, R^{1}=\mathrm{CH}_{3}\end{array}$ & $\begin{array}{l}\text { 2a: } \mathrm{R}^{3}=\mathrm{H} ; \\
\text { 2b: } \mathrm{R}^{3}=\mathrm{CH}_{2} \mathrm{CH}_{3} ; \\
\text { 2c: } \mathrm{R}^{3}=\mathrm{CH}_{3}\end{array}$ \\
\hline
\end{tabular}

$$
\begin{aligned}
& \text { 3a: } R=R^{1}=R^{2}=R^{3}=H(70 \%) \text {; } \\
& \text { 3b: } \mathrm{R}=\mathrm{CH}_{3}, \mathrm{R}^{1}=\mathrm{R}^{2}=\mathrm{R}^{3}=\mathrm{H}(72 \%) \text {; } \\
& \text { 3c: } \mathrm{R}=\mathrm{R}^{3}=\mathrm{H}, \mathrm{R}^{1}=\mathrm{R}^{2}=\mathrm{CH}_{3}(79 \%) \text {; } \\
& \text { 3d: } \mathrm{R}=\mathrm{Cl}, \mathrm{R}^{1}=\mathrm{R}^{2}=\mathrm{R}^{3}=\mathrm{H}(71 \%) \text {; } \\
& \text { 3e: } R=\mathrm{NO}_{2}, \mathrm{R}^{1}=\mathrm{R}^{2}=\mathrm{R}^{3}=\mathrm{H}(65 \%) \text {; } \\
& \text { 3f: } R=B r, R^{1}=R^{2}=R^{3}=H(73 \%) \text {; } \\
& \text { 3g: } \mathrm{R}=\mathrm{H}, \mathrm{R}^{1}=\mathrm{R}^{2}=\mathrm{CH}_{3}, \mathrm{R}^{3}=\mathrm{CH}_{2} \mathrm{CH}_{3}(79 \%) \text {; } \\
& \text { 3h: } \mathrm{R}=\mathrm{Cl}, \mathrm{R}^{1}=\mathrm{R}^{2}=\mathrm{H}, \mathrm{R}^{3}=\mathrm{CH}_{2} \mathrm{CH}_{3}(73 \%) \text {; } \\
& \text { 3i: } \mathrm{R}=\mathrm{NO}_{2}, \mathrm{R}^{1}=\mathrm{R}^{2}=\mathrm{H}, \mathrm{R}^{3}=\mathrm{CH}_{2} \mathrm{CH}_{3}(67 \%) \text {; } \\
& \text { 3j: } \mathrm{R}=\mathrm{Br}, \mathrm{R}^{1}=\mathrm{R}^{2}=\mathrm{H}, \mathrm{R}^{3}=\mathrm{CH}_{2} \mathrm{CH}_{3}(76 \%) \text {; } \\
& \text { 3k: } \mathrm{R}=\mathrm{R}^{2}=\mathrm{H}, \mathrm{R}^{1}=\mathrm{R}^{3}=\mathrm{CH}_{3}(78 \%) \text {; } \\
& \text { 3I: } \mathrm{R}=\mathrm{H}, \mathrm{R}^{1}=\mathrm{R}^{2}=\mathrm{R}^{3}=\mathrm{CH}_{3}(81 \%) \text {; } \\
& \text { 3m: } \mathrm{R}=\mathrm{Cl}, \mathrm{R}^{1}=\mathrm{R}^{2}=\mathrm{H}, \mathrm{R}^{3}=\mathrm{CH}_{3}(72 \%) \text {; } \\
& \text { 3n: } \mathrm{R}=\mathrm{NO}_{2}, \mathrm{R}^{1}=\mathrm{R}^{2}=\mathrm{H}, \mathrm{R}^{3}=\mathrm{CH}_{3}(67 \%) \text {; } \\
& \text { 3o: } R=B r, R^{1}=R^{2}=H, R^{3}=C_{3}(74 \%)
\end{aligned}
$$

Scheme 1. Synthesis of pyrazoles 3a-o by the reaction of amidrazones 1a-g with 2a-c.<smiles>N#Cc1cnn(C(=Nc2ccc(Br)cc2)c2ccccc2)c1N</smiles>

Figure 1. Positional designations for compounds 3 .

Compound 3c illustrates the method. Mass and elemental analyses indicated that the molecular weight is consistent with the molecular formula $\mathrm{C}_{19} \mathrm{H}_{17} \mathrm{~N}_{4}$. The IR spectra showed absorbance for amino, nitrile and $\mathrm{C}=\mathrm{N}$ groups at $v_{\max }\left(\mathrm{cm}^{-1}\right)=3384-3265\left(\mathrm{NH}_{2}\right), 2217(\mathrm{CN}), 1651,1610(\mathrm{C}=\mathrm{N})$. For assigning the NMR spectra, the 2,6-dimethylphenyl ring provides a convenient starting point. The methyl protons and their attached carbon are distinctive at $\delta_{H}=2.05$ and $\delta_{C}=18.3$ respectively. The methyl carbon gives HMBC correlation with a $2 \mathrm{H}$ doublet at $\delta_{H}=6.92$, assigned as H- $m$ '; the attached carbons appear at $\delta_{C}=127.7$. H- $m$ ' gives COSY correlation with a $1 \mathrm{H}$ triplet at $\delta_{H}=6.81$, assigned as H- $p$ '; the attached carbon appears at $\delta_{C}=123.5$. $\mathrm{H}-m$ ', H- $p$ ', and $\mathrm{CH}_{3}$ all give $\mathrm{HMBC}$ 
correlation with a carbon at $\delta_{C}=144.2$, assigned as $\mathrm{H}-i^{\prime}$ : this is a three-bond coupling from $\mathrm{CH}_{3}$ and $\mathrm{H}-m$ ', and a four-bond coupling from H- $p^{\prime}$. H-p ' gives HMBC correlation with a carbon at $\delta_{C}$ $=126.6$, assigned as C-o'; this is a three-bond coupling. C-o' gives no other HMBC correlation; this signal is the only tall carbon signal lacking attached protons. (The ${ }^{13} \mathrm{C}$ lines from doublydegenerate carbons are about twice the height of the others: four signals when both phenyl rings are twofold symmetric, two signals in 3k). The protons of the monosubstituted phenyl ring barely separate into a $1 \mathrm{H}$ triplet at $\delta_{H}=7.33$, a $2 \mathrm{H}$ "triplet" at $\delta_{H}=7.27$, and a $2 \mathrm{H}$ doublet at $\delta_{H}=7.19$; these are assigned as $\mathrm{H}-p, \mathrm{H}-m$, and $\mathrm{H}-o$ respectively. The attached carbons appear at $\delta_{C}=130.0$ $(\mathrm{C}-p), 128.0(\mathrm{C}-m)$, and $128.2(\mathrm{C}-o)$. H- $m$ gives HMBC correlation with a non-protonated carbon at $\delta_{C}=131.2$, assigned as $\mathrm{C}-i$; this is a three-bond coupling.

Table 1. NMR assignments of compound 3c

\begin{tabular}{|c|c|c|c|}
\hline${ }^{1}$ H NMR (DMSO-d $\left.d_{6}\right)$ & \multicolumn{2}{|c|}{${ }^{1} \mathrm{H}-{ }^{1} \mathrm{H} \mathrm{COSY}$} & Assignment \\
\hline $8.20(\mathrm{bs} ; 2 \mathrm{H})$ & & & $\mathrm{NH}_{2}$ \\
\hline $7.74(\mathrm{~s} ; 1 \mathrm{H})$ & & & $\mathrm{H}-3$ \\
\hline $7.33(\mathrm{t}, J 7.3 ; 1 \mathrm{H})$ & $7.27,7.1$ & & $\mathrm{H}-p$ \\
\hline $7.27(\mathrm{dd}, J 7.7,7.2 ; 2 \mathrm{H})$ & $7.33,7.1$ & & $\mathrm{H}-m$ \\
\hline $7.19(\mathrm{~d}, J 7.3 ; 2 \mathrm{H})$ & $7.33,7.2$ & & $\mathrm{H}-\mathrm{O}$ \\
\hline $6.92(\mathrm{~d}, J 7.5 ; 2 \mathrm{H})$ & 6.81 & & $\mathrm{H}-m$ \\
\hline $6.81(\mathrm{t}, J 7.3 ; 1 \mathrm{H})$ & 6.92 & & H- $p$ \\
\hline $2.04(\mathrm{~s} ; 6 \mathrm{H})$ & & & $\mathrm{Ar}-\mathrm{CH}_{3}$ \\
\hline${ }^{13} \mathrm{C}$ NMR (DMSO- $\left.d_{6}\right)$ & HSQC & HMBC & Assignment \\
\hline 156.2 & & $7.27,7.19,2.04$ & $C-\alpha$ \\
\hline 154.6 & & 7.74 & C-5 \\
\hline 144.2 & & $6.92,6.81,2.04$ & $\mathrm{C}-i$ \\
\hline 141.7 & 7.74 & & C-3 \\
\hline 131.2 & & 7.27 & $\mathrm{C}-i$ \\
\hline 130.0 & 7.33 & 7.19 & C- $p$ \\
\hline 128.2 & 7.19 & $7.33,7.19$ & $\mathrm{C}-\mathrm{O}$ \\
\hline 128.0 & 7.27 & $7.27,2.04$ & $\mathrm{C}-m$ \\
\hline 127.7 & 6.92 & & $\mathrm{C}-m$ \\
\hline 126.6 & & 6.81 & $\mathrm{C}-\mathrm{o}$ \\
\hline 123.5 & 6.81 & & C- $p{ }^{\prime}$ \\
\hline 114.2 & & 7.74 & $\mathrm{C}-4 \mathrm{a}$ \\
\hline 72.9 & & $8.20,7.74$ & $\mathrm{C}-4$ \\
\hline 18.3 & 2.18 & $6.92,6.81$ & $\mathrm{Ar}-\mathrm{CH}_{3}$ \\
\hline${ }^{15} \mathrm{~N}$ NMR (DMSO-d $)$ & HSQC & НMBC & Assignment \\
\hline-94.9 & & 7.74 & $\mathrm{~N}-2$ \\
\hline-178.0 & & $8.20,7.74$ & $\mathrm{~N}-1$ \\
\hline-310.4 & 8.20 & & $\mathrm{NH}_{2}$ \\
\hline
\end{tabular}


This leaves the pyrazole ring. The amino protons give a broadened singlet at $\delta_{H}=8.20$, whose attached nitrogen appears at $\delta_{N}=70.0$. The amino protons give HMBC correlation with a carbon at $\delta_{C}=72.9$, assigned as C-4. (The upfield shift arises because this carbon is part of a push-pull system. ${ }^{24}$ ) C-4 also gives HMBC correlation with a singlet at $\delta_{H}=7.74$, assigned as $\mathrm{H}-3$; its attached carbon appears at $\delta_{C}=141.7$. H-3 also gives HMBC correlation with a carbon at $\delta_{C}=$ 154.6, assigned as $\mathrm{C}-5$, and with the nitrile carbon at $\delta_{C}=114.2$. $\mathrm{H}-3$ gives HMBC correlation with a nitrogen at $\delta_{N}=-178.0$; this nitrogen also gives HMBC correlation with $\mathrm{NH}_{2}$. These are both three-bond correlations if this nitrogen is $\mathrm{N}-1$, which is also consistent with its chemical shift (between the normal $s p^{3}$ and $s p^{2}$ ranges). H-3 also gives HMBC correlation with a nitrogen at $\delta_{N}$ $=-94.9$, which is assigned as $\mathrm{N}-2$; this is a two-bond correlation, but two-bond $\mathrm{H}-\mathrm{N}$ couplings are larger than four-bond couplings (like that between $\mathrm{H}-3$ and the nitrile nitrogen, which is not observed). The remaining carbon, at $\delta_{C}=156.2$, is assigned as C- $\alpha$ based on chemical shift; it gives $\mathrm{HMBC}$ correlation with $\mathrm{H}-m$ (four bonds), $\mathrm{H}-o$ (three bonds), and $\mathrm{CH}_{3}$ (five bonds). Table 1 summarizes the NMR assignments of compound $\mathbf{3 c}$.

The regiochemical assignment depends on the ${ }^{1} \mathrm{H}^{-15} \mathrm{~N}$ correlations. Nitrogen signals were measured for thirteen of the fifteen products. Nitrogen signals were observed indirectly via the proton channel, using gradient-enhanced HSQC and HMBC experiments; ${ }^{25}$ HMBC experiments utilized a standard mixing time of $65 \mathrm{~ms}$; this is supposed to equal $1 /[2 \mathrm{~J}(\mathrm{~N}, \mathrm{H})]$, corresponding to $J 7.7 \mathrm{~Hz}$. Because ${ }^{1} \mathrm{H}-{ }^{15} \mathrm{~N}$ coupling constants are smaller than the corresponding ${ }^{1} \mathrm{H}-{ }^{13} \mathrm{C}$ couplings, ${ }^{26}$ the intensity and observability of cross-peaks are sensitive to the mixing time,${ }^{27}$ and we could not detect all the nitrogen atoms; however, the signals detected suffice to prove the regiochemistry. Table 2 lists the observed ${ }^{15} \mathrm{~N}$ signals, together with the protons to which they give HSQC or HMBC correlations; Figure 2 shows the general structures of products 3 and possible regioisomers 4 .

Table 2. Nitrogen chemical shifts of products, and their ${ }^{1} \mathrm{H}$ correlations

\begin{tabular}{|c|c|c|c|c|c|}
\hline Compound & $\mathbf{N H}_{2}{ }^{\mathrm{a}}$ & $\mathrm{N}-1^{\mathrm{b}}$ & $\mathrm{N}-2^{\mathrm{b}}$ & $\mathrm{N}-\beta^{\mathrm{b}}$ & $\mathrm{NO}_{2}{ }^{\mathrm{b}}$ \\
\hline $\mathbf{3 a}$ & $-310.7\left(\mathrm{NH}_{2}\right)$ & $-177.7(\mathrm{H}-3)$ & $\mathrm{n} / \mathrm{o}^{\mathrm{c}}$ & $\mathrm{n} / \mathrm{o}$ & $--^{d}$ \\
\hline $3 \mathbf{b}$ & $-310.8\left(\mathrm{NH}_{2}\right)$ & $-177.3\left(\mathrm{NH}_{2}, \mathrm{H}-3\right)$ & $-94.7(\mathrm{H}-3)$ & $-101.6\left(\mathrm{H}-O^{\prime}\right)$ & -- \\
\hline $3 c$ & $-310.4\left(\mathrm{NH}_{2}\right)$ & $-178.0\left(\mathrm{NH}_{2}, \mathrm{H}-3\right)$ & $-94.9(\mathrm{H}-3)$ & $\mathrm{n} / \mathrm{o}$ & -- \\
\hline 3d & $-310.2\left(\mathrm{NH}_{2}\right)$ & $\mathrm{n} / \mathrm{o}$ & $\mathrm{n} / \mathrm{o}$ & $\mathrm{n} / \mathrm{o}$ & -- \\
\hline $3 f$ & $-309.6\left(\mathrm{NH}_{2}\right)$ & $-177.5\left(\mathrm{NH}_{2}, \mathrm{H}-3\right)$ & $-95.0(\mathrm{H}-3)$ & $-104.5\left(\mathrm{H}-O^{\prime}\right)$ & -- \\
\hline $3 g$ & $-310.5\left(\mathrm{NH}_{2}\right)$ & $\mathrm{n} / \mathrm{o}$ & $-102.2(\mathrm{H}-3 \mathrm{a})^{\mathrm{e}}$ & $\mathrm{n} / \mathrm{o}$ & -- \\
\hline $3 \mathbf{i}$ & $-310.5\left(\mathrm{NH}_{2}\right)$ & $\mathrm{n} / \mathrm{o}$ & $-102.7(\mathrm{H}-3 \mathrm{a})$ & $-106.8\left(\mathrm{H}-o^{\prime}\right)$ & $-10.2\left(\mathrm{H}-m^{\prime}\right)$ \\
\hline 3j & $-309.9\left(\mathrm{NH}_{2}\right)$ & $\mathrm{n} / \mathrm{o}$ & $-102.6(\mathrm{H}-3 \mathrm{a})$ & $\mathrm{n} / \mathrm{o}$ & -- \\
\hline $3 \mathbf{k}$ & $-310.1\left(\mathrm{NH}_{2}\right)$ & $-181.6\left(\mathrm{NH}_{2}\right)$ & $-101.6(\mathrm{H}-3 \mathrm{a})$ & $-103.2\left(H-3^{\prime}\right)^{f}$ & -- \\
\hline 31 & $-310.4\left(\mathrm{NH}_{2}\right)$ & $-182.4\left(\mathrm{NH}_{2}\right)$ & $-101.4(\mathrm{H}-3 \mathrm{a})$ & $\mathrm{n} / \mathrm{o}$ & -- \\
\hline $3 \mathrm{~m}$ & $-309.5\left(\mathrm{NH}_{2}\right)$ & $-181.5\left(\mathrm{NH}_{2}\right)$ & $-101.9(\mathrm{H}-3 \mathrm{a})$ & $-107.1\left(\mathrm{H}-o^{\prime}\right)$ & -- \\
\hline $3 n$ & $-308.6\left(\mathrm{NH}_{2}\right)$ & $\mathrm{n} / \mathrm{o}$ & $-101.9(\mathrm{H}-3 \mathrm{a})$ & $-107.1\left(\mathrm{H}-O^{\prime}\right)$ & $\mathrm{n} / \mathrm{o}$ \\
\hline 30 & $-309.5\left(\mathrm{NH}_{2}\right)$ & $-181.6\left(\mathrm{NH}_{2}\right)$ & $-101.8(\mathrm{H}-3 \mathrm{a})$ & $-106.6\left(\mathrm{H}-o^{\prime}\right)$ & -- \\
\hline
\end{tabular}


${ }^{a}$ HSQC correlation. b: HMBC correlation. c: Not observed. d: No such nitrogen. ${ }^{\mathrm{e}}$ Pseudobenzylic methyl or methylene protons attached to C-3. ${ }^{\mathrm{f}}$ Non-symmetric ring: same position as $\mathrm{H}-\mathrm{o}^{\prime}$ in other rings.
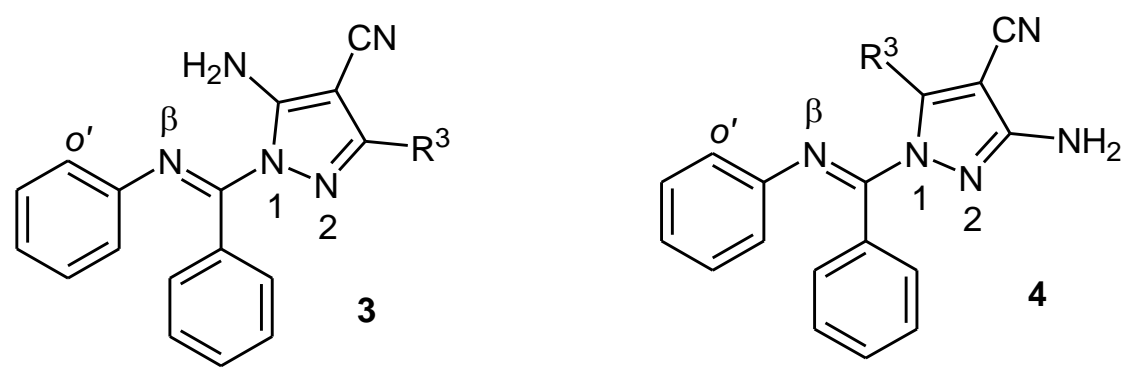

Figure 2. General structures of products 3 and possible regioisomers 4.

In all structures, the broadened amino protons give HSQC correlation with an $s p^{3}$ nitrogen, which is assigned as $\mathbf{N H}_{2}$. In seven of the products, the same broadened protons also give HMBC correlation with a nitrogen at $\delta_{N}=-177$ to -183 ; this chemical shift corresponds to the formally $s p^{3}$ nitrogen of an amidine, ${ }^{28}$ and the signal is assigned as $\mathrm{N}-1$, consistent with structure $\mathbf{3}$. In the regioisomer $\mathbf{4}$, the nitrogen three bonds from the amino protons would be $s p^{2}$ hybridized, and would have a chemical shift of approximately $\delta_{N}=-110$. Consistent with the assignment of structure 3 is the HMBC correlation observed, in products $\mathbf{3 g - o ,}$, between $\mathrm{H}-3 \mathrm{a}$ and an $s p^{2}$ nitrogen at $\delta_{N}=-101$ to -103 , assigned as $\mathrm{N}-2$. No nitrile nitrogen atoms were observed: they are all at least four bonds from the nearest protons. The presence of the nitrile groups is deduced from observations of the nitrile carbons in the ${ }^{13} \mathrm{C}$ NMR spectra, and of nitrile absorptions in the IR spectra.

Mechanistically, the reaction can be described (Scheme 2) as due nucleophilic attack of the hydrazine group to $\mathrm{C}-1$ to form salt $\mathbf{A}$.

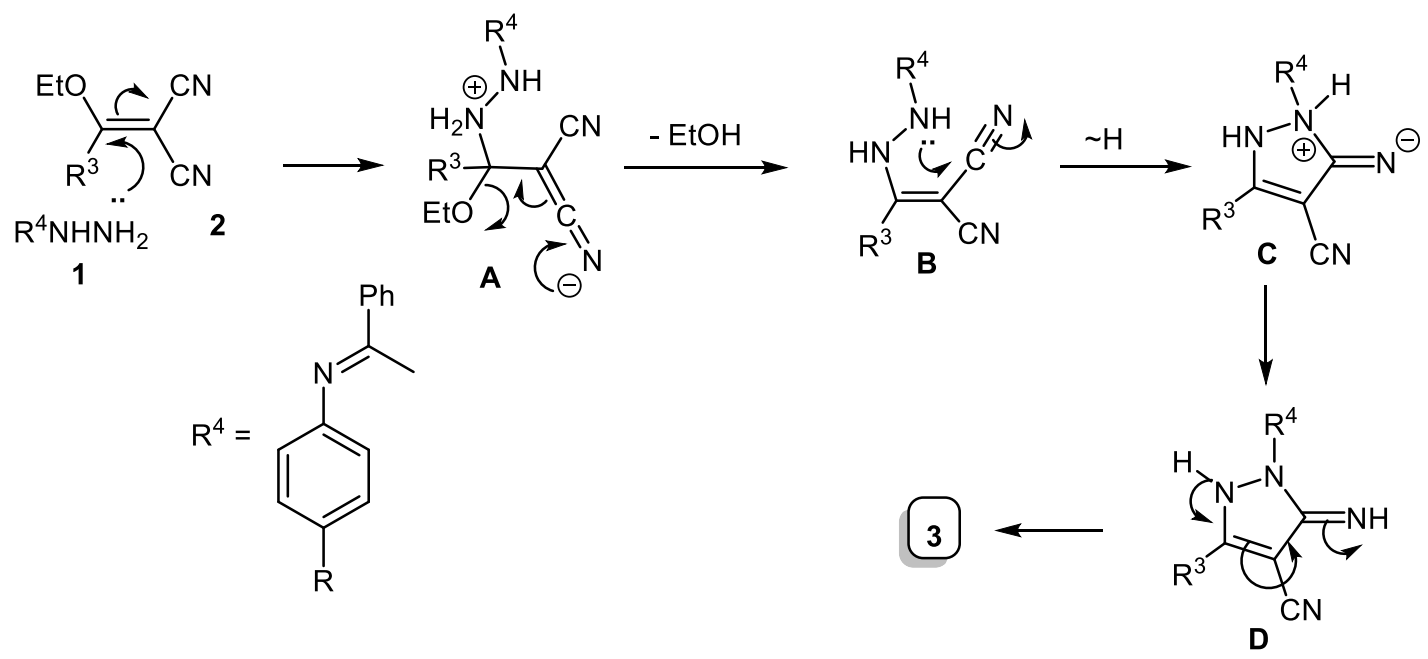

Scheme 2. Mechanism of pyrazole formation from reaction of 1a-g with 2a-c 
The observation of products $\mathbf{3}$, rather than $\mathbf{4}$, requires that this attack occur at $\mathrm{C}-1$ instead of one of the nitrile carbons. Elimination of ethanol would then give intermediate B. Subsequently, internal cyclization of $\mathbf{B}$ would give $\mathbf{C}$, which would be in tautomerism with $\mathbf{D}$ and ultimately give product $\mathbf{3}$ after proton transfer.

\section{Experimental Section}

General. NMR spectra were measured on a Bruker AV-400 spectrometer (Bruker BioSpin Corp., Billerica, MA, USA) (400 MHz for ${ }^{1} \mathrm{H}, 100 \mathrm{MHz}$ for ${ }^{13} \mathrm{C}$, and $40.55 \mathrm{MHz}$ for ${ }^{15} \mathrm{~N}$ ) at Florida Institute of Technology, USA. The ${ }^{1} \mathrm{H}$ and ${ }^{13} \mathrm{C}$ chemical shifts are given relative to internal standard TMS $=0 ;{ }^{15} \mathrm{~N}$ shifts are reported versus external neat nitromethane $=0 .{ }^{29}$ For preparative thin layer chromatography (PLC), glass plates $(20 \times 48 \mathrm{~cm})$ were covered with a slurry of silica gel Merck PF254 and air dried and developed using the solvents listed. Zones were detected by quenching of indicator fluorescence upon exposure to $254 \mathrm{~nm}$ UV light. Elemental analyses were carried in the National Research center, Dokki, Cairo, Egypt. Mass spectrometry was performed by electron impact at $70 \mathrm{eV}$, with a Finnigan Mat 8430 spectrometer in the National Research center, Dokki, Cairo, Egypt. IR spectra using KBr pellets, were run on a FT-IR (Bruker), Minia University, El-Minia, Egypt. Amidrazones 1a-g were prepared according to reference 23.

\section{Reaction of amidrazones 1a-g with 2a-c (Scheme 1)}

General procedure. A $100 \mathrm{~mL}$ round-bottom flask was flame-dried. Dry absolute ethyl alcohol $(20 \mathrm{~mL})$ containing a few drops of triethylamine $\mathrm{Et}_{3} \mathrm{~N}$ and $1 \mathrm{~mL}$ of DMF, a mixture of 1a-g (1 $\mathrm{mmol}$ ) and 2a-c (1 mmol) was added. The mixture was stirred under reflux for $2-10 \mathrm{~h}$ (the reaction was followed by TLC analysis). The solvent was then removed under vacuum and the residue was separated. The products 3a-o were obtained and were recrystallized from the stated solvents.

(E)-5-Amino-1-(phenyl(phenylimino)methyl)-1H-pyrazole-4-carbonitrile (3a). Pale yellow crystals (EtOH), yield: $0.23 \mathrm{~g}(70 \%))$; mp 204-205 ${ }^{\circ} \mathrm{C}$. IR $\left(\mathrm{KBr} ; v_{\max }, \mathrm{cm}^{-1}\right): 3369-3273\left(\mathrm{NH}_{2}\right)$, $3057\left(\mathrm{CH}\right.$ aromatic), $2218(\mathrm{C} \equiv \mathrm{N}), 1644,1618(\mathrm{C}=\mathrm{N}), 1552(\mathrm{C}=\mathrm{C}) .{ }^{1} \mathrm{H}$ NMR (DMSO- $\left.d_{6}\right): \delta_{H} 8.25$ (bs, 2H; $\mathrm{NH}_{2}$ ), 7.75 (s, 1H; H-3), 7.31 (m, 5H; H-o,m,p), 7.19 (t, J 7.4 Hz, 2H; H-m), 6.98 (t, 1H, $J 7.2 \mathrm{~Hz} ; \mathrm{H}-p$ ), 6.82 (d, 2H, $J 7.6 \mathrm{~Hz} ; \mathrm{H}-o$ ). ${ }^{13} \mathrm{C}$ NMR (DMSO- $\left.d_{6}\right): \delta_{C} 157.7(\mathrm{C}-\alpha), 154.4(\mathrm{C}-5)$, 146.4 (C-i'), 141.9 (C-3), 130.8 (C-i), 129.4 (C-p), 129.2 (2C; C-o), 128.5 (2C; C-m'), 127.6 (2C; C-m), $123.8\left(\mathrm{C}-p^{\prime}\right), 121.5\left(2 \mathrm{C}\right.$; C-o'), $114.2(\mathrm{C} \equiv \mathrm{N}), 72.7(\mathrm{C}-4) .{ }^{15} \mathrm{~N}$ NMR: Table 2. MS (EI, 70 $\mathrm{eV}): m / z$ (\%) $287\left(\mathrm{M}^{+}, 10\right), 180$ (100), 77 (59). Anal. Calcd for $\mathrm{C}_{17} \mathrm{H}_{13} \mathrm{~N}_{5}$ (287.12): C, 71.06; H, 4.56; N, 24.37. Found: C, 70.90; H, 4.49; N, 24.30.

(E)-5-Amino-1-(phenyl(p-tolylimino)methyl)-1H-pyrazole-4-carbonitrile (3b). Pale yellow crystals (EtOH), yield: $0.23 \mathrm{~g}(77 \%) ; \mathrm{mp} 241-242{ }^{\circ} \mathrm{C}$. IR $\left(\mathrm{KBr} ; v_{\max }, \mathrm{cm}^{-1}\right): 3438-3294\left(\mathrm{NH}_{2}\right), 3061$ (CH aromatic), 2921 (CH aliphatic), $2217(\mathrm{C} \equiv \mathrm{N}), 1650,1607(\mathrm{C}=\mathrm{N}), 1548(\mathrm{C}=\mathrm{C}) .{ }^{1} \mathrm{H}$ NMR (DMSO-d $): \delta_{H} 8.22$ (bs, 2H; NH$)_{2}, 7.71$ (s, 1H; H-3), 7.30 (m, 3H; H-p, m), 7.26 (m, 2H; H-o), $6.96\left(\mathrm{~d}, J 7.8 \mathrm{~Hz}, 2 \mathrm{H} ; \mathrm{H}-m\right.$ '), 6.68 (d, J 7.7 Hz, 2H; H-o'), 2.18 (s, 3H; $\left.\mathrm{CH}_{3}\right) .{ }^{13} \mathrm{C}$ NMR (DMSO- 
$\left.d_{6}\right): \delta_{C} 157.6(\mathrm{C}-\alpha), 154.4(\mathrm{C}-5), 143.8\left(\mathrm{C}-i^{\prime}\right), 141.8(\mathrm{C}-3), 132.8\left(\mathrm{C}-p^{\prime}\right), 130.9(\mathrm{C}-i), 129.4(\mathrm{C}-p)$, 129.2 (2C; C-o), 129.0 (2C; C-m'), 127.6 (2C; C-m), 121.5 (2C; C-o'), $114.2(\mathrm{C} \equiv \mathrm{N}), 72.7$ (C-4), $20.3\left(\mathrm{CH}_{3}\right) .{ }^{15} \mathrm{~N}$ NMR: Table 2. MS (EI, $\left.70 \mathrm{eV}\right): m / z(\%) 301\left(\mathrm{M}^{+}, 10\right), 238$ (100), 214 (80), 194 (30), 135 (67), 77 (50). Anal. Calcd for $\mathrm{C}_{18} \mathrm{H}_{15} \mathrm{~N}_{5}$ (301.13): C, 71.74; H, 5.02; N, 23.24. Found: C, 71.60; H, 5.00; N, 23.10.

\section{(E)-5-Amino-1-((2',6'-dimethylphenyl)imino)(phenyl)methyl)-1H-pyrazole-4-carbonitrile}

(3c). Pale yellow crystals $\left(\mathrm{CH}_{3} \mathrm{OH} / \mathrm{DCM}\right)$, yield: $0.25 \mathrm{~g}(79 \%) ; \mathrm{mp} 247-248^{\circ} \mathrm{C}$. IR ( $\mathrm{KBr} ; v_{\max }, \mathrm{cm}^{-}$ 1) 3384-3265 $\left(\mathrm{NH}_{2}\right), 3112(\mathrm{CH}$ aromatic), $2947(\mathrm{CH}$ aliphatic $), 2217(\mathrm{C} \equiv \mathrm{N}), 1651,1610(\mathrm{C}=\mathrm{N})$, $1548(\mathrm{C}=\mathrm{C})$. NMR (DMSO-d6): Tables 1 and 2. MS (EI, $70 \mathrm{eV}): 315\left(\mathrm{M}^{+}, 45\right), 207(100), 153$ (42), 136 (27), 106 (8). Calcd for $\mathrm{C}_{19} \mathrm{H}_{17} \mathrm{~N}_{5}$ (315.15): C, 72.36; H, 5.43; N, 22.21. Found: C, 72.20; $\mathrm{H}, 5.30 ; \mathrm{N}, 22.10$.

(E)-5-Amino-1-((4'-chlorophenylimino)(phenyl)methyl)-1H-pyrazole-4-carbonitrile $\quad(3 d)$. Pale yellow crystals (EtOH), yield: $0.22 \mathrm{~g}(71 \%)$; mp 258-259 ${ }^{\circ} \mathrm{C}$. IR $\left(\mathrm{KBr} ; v_{\max }, \mathrm{cm}^{-1}\right)$ : $3378-$ 3276( $\left.\mathrm{NH}_{2}\right), 3054\left(\mathrm{CH}\right.$ aromatic), $2220(\mathrm{C} \equiv \mathrm{N}), 1642,1618(\mathrm{C}=\mathrm{N}), 1550(\mathrm{C}=\mathrm{C}) .{ }^{1} \mathrm{H}$ NMR (DMSO$\left.d_{6}\right): \delta_{H} 8.22\left(\mathrm{~s}, 2 \mathrm{H} ; \mathrm{NH}_{2}\right), 7.73(\mathrm{~s}, 1 \mathrm{H} ; \mathrm{H}-3), 7.31$ (m, 5H; H-o,m,p), 7.22 (d, J 8.2 Hz, 2H; H-m '), $6.82(\mathrm{~d}, J 8.0 \mathrm{~Hz}, 2 \mathrm{H}$; H-o $) .{ }^{13} \mathrm{C}$ NMR (DMSO- $\left.d_{6}\right): \delta_{C} 158.2(\mathrm{C}-\alpha), 154.4(\mathrm{C}-5), 145.5\left(\mathrm{C}-i^{\prime}\right)$, 142.1 (C-3), 130.6 (C-i), 129.5 (C-p), 129.2 (2C; C-o), 128.4 (C-p'), 128.4 (2C; C-m'), $127.6(2 \mathrm{C}$; C-m), $123.4\left(2 \mathrm{C}\right.$; C-o'), $114.2(\mathrm{C} \equiv \mathrm{N}), 72.7(\mathrm{C}-4) .{ }^{15} \mathrm{~N}$ NMR: Table 2. MS (EI, $\left.70 \mathrm{eV}\right): \mathrm{m} / \mathrm{z}(\%)$ $323(\mathrm{M}+2,8), 321\left(23, \mathrm{M}^{+}\right), 214$ (100), 111 (12), 75 (13). Anal. Calcd for $\mathrm{C}_{17} \mathrm{H}_{12} \mathrm{ClN}_{5}(321.08$ ): C, 63.46; H, 3.76; N, 21.77. C, 63.34; H, 3.65; N, 10.90 .

(E)-5-Amino-1-((4'-nitrophenylimino)(phenyl)methyl)-1H-pyrazole-4-carbonitrile (3e). Pale yellow crystals $\left(\mathrm{CH}_{3} \mathrm{OH} / \mathrm{DCM}\right)$, yield: $0.23 \mathrm{~g}(71 \%)$; $\mathrm{mp} 270-271{ }^{\circ} \mathrm{C}$. IR $\left(\mathrm{KBr} ; v_{\max }, \mathrm{cm}^{-1}\right)$ : $3453-$ $3295\left(\mathrm{NH}_{2}\right), 3095(\mathrm{CH}$ aromatic $), 2217(\mathrm{C} \equiv \mathrm{N}), 1659,1610(\mathrm{C}=\mathrm{N}), 1508(\mathrm{C}=\mathrm{C}) .{ }^{1} \mathrm{H} \mathrm{NMR}\left(\mathrm{CDCl}_{3}\right)$ : $\delta_{H} 8.05$ (bs, 2H, NH ), 7.5 (s, 1H, H-3), 7.30-7.20 (m, 5H, H-o,m,p), 6.90-6.88 (d, J 7.5 Hz, 2H; H-m '), 6.80-6.77 (d, J 7.5 Hz, 2H; H-o '). ${ }^{13} \mathrm{C} \mathrm{NMR}\left(\mathrm{CDCl}_{3}\right): \delta_{C} 159.1,154.6,152.2,144.3,142.1$, 130.9, 129.4, 129.2 (2C), 128.3 (2C), 124.7 (2C), 122.3 (2C), 113.3, 75.4. MS (EI, $70 \mathrm{eV}): \mathrm{m} / z$ (\%) $332\left(\mathrm{M}^{+}, 25\right), 225$ (100), 179 (37), 76 (53). Anal. Calcd for $\mathrm{C}_{17} \mathrm{H}_{12} \mathrm{~N}_{6} \mathrm{O}_{2}$ (332.10): C, 61.44; $\mathrm{H}, 3.64 ; \mathrm{N}, 25.29$

(E)-5-Amino-1-((4'-bromophenylimino)(phenyl)methyl)-1H-pyrazole-4-carbonitrile (3f). Pale yellow crystals $\left(\mathrm{CH}_{3} \mathrm{OH} / \mathrm{DCM}\right)$, yield: $0.25 \mathrm{~g}(70 \%)$; mp 280-281 ${ }^{0} \mathrm{C}$. IR $\left(\mathrm{KBr} ; v_{\max }, \mathrm{cm}^{-1}\right)$ : 3382-3278 $\left(\mathrm{NH}_{2}\right), 3100\left(\mathrm{CH}\right.$ aromatic), $2220(\mathrm{C} \equiv \mathrm{N}), 1642,1616(\mathrm{C}=\mathrm{N}), 1549(\mathrm{C}=\mathrm{C}) .{ }^{1} \mathrm{H}$ NMR (DMSO-d6): $\delta_{H} 8.21\left(\mathrm{bs}, 2 \mathrm{H} ; \mathrm{NH}_{2}\right), 7.74(\mathrm{~s}, 1 \mathrm{H} ; \mathrm{H}-3), 7.36(\mathrm{~m}, 2 \mathrm{H} ; \mathrm{H}-m$ '), 7.34 (m, 1H; H-p), 7.33 (m, 2H; H-o/m), 7.29 (m, 2H; H-m/o), 6.77 (d, $J 8.6 \mathrm{~Hz}, 2 \mathrm{H} ; \mathrm{H}-o$ '). ${ }^{13} \mathrm{C}$ NMR (DMSO- $\left.d_{6}\right)$ : $\delta_{C} 158.1(\mathrm{C}-\alpha), 154.4(\mathrm{C}-5), 145.9\left(\mathrm{C}-i^{\prime}\right), 142.1(\mathrm{C}-3), 131.3$ (2C; C-m'), $130.6\left(\mathrm{C}-i^{\prime}\right), 129.6(\mathrm{C}-$ p), $129.2(2 \mathrm{C}$; C-m/o), 127.7 (2C; C-o/m), $123.8(2 \mathrm{C}$; C-o'), $116.1(\mathrm{C}-p$ '), $114.2(\mathrm{C} \equiv \mathrm{N}), 72.7(\mathrm{C}-$ 4). ${ }^{15} \mathrm{~N}$ NMR: Table 2. MS (EI, $\left.70 \mathrm{eV}\right): 367(\mathrm{M}+2,8), 365$ (6, $\left.\mathrm{M}^{+}\right), 306(36), 288$ (17), 245 (14), 153 (100), 136 (74), 119 (11), 106 (16). Anal. Calcd for $\mathrm{C}_{17} \mathrm{H}_{12} \mathrm{BrN}_{5}$ (365.03): C, 55.75; H, 3.30; N, 19.12. C, 55.60; H, 3.15; N, 19.00. Found: C, 55.60; H, 3.20; N, 19.00.

(E)-5-Amino-1-(((2',6'-dimethylphenyl)imino)(phenyl)methyl)-3-ethyl-1H-pyrazole-4carbonitrile (3g). Pale yellow crystals (EtOAc), yield: $0.25 \mathrm{~g}(74 \%)$; mp 180-182 ${ }^{\circ} \mathrm{C}$. IR (KBr; 
$\left.v_{\max }, \mathrm{cm}^{-1}\right): 3366-3289\left(\mathrm{NH}_{2}\right), 3055$ (CH aromatic), $2967(\mathrm{CH}$ aliphatic), $2214(\mathrm{C} \equiv \mathrm{N}), 1651,1618$ $(\mathrm{C}=\mathrm{N}), 1553(\mathrm{C}=\mathrm{C}) .{ }^{1} \mathrm{H}$ NMR (DMSO-d $): \delta_{H} 8.16\left(\mathrm{bs}, 2 \mathrm{H} ; \mathrm{NH}_{2}\right), 7.33(\mathrm{t}, J 7.3 \mathrm{~Hz}, 1 \mathrm{H} ; \mathrm{H}-p), 7.26$ $(\mathrm{dd}, J$ 7.7, 7.2 Hz, 2H; H-m), 7.18 (d, J 7.2, 2H; H-o), 6.91 (d, J 7.5 Hz, 2H; H-m'), 6.80 (t, J 7.5 $\mathrm{Hz}, 1 \mathrm{H} ; \mathrm{H}-p$ '), 2.45 (q, J 7.6, 2H; $\left.\mathrm{CH}_{2}\right), 2.05$ (s, 6H; $\left.\mathrm{Ar}-\mathrm{CH}_{3}\right), 1.11\left(\mathrm{t}, J 7.6 \mathrm{~Hz}, 3 \mathrm{H} ; \mathrm{CH}_{2} \mathrm{CH}_{3}\right) .{ }^{13} \mathrm{C}$ NMR (DMSO- $\left.d_{6}\right): \delta_{C} 156.0(\mathrm{C}-\alpha), 155.2,155.0(\mathrm{C}-3,5), 144.3(\mathrm{C}-i$ ') $131.2(\mathrm{C}-i), 130.0(\mathrm{C}-p)$, 128.3 (2C; C-o), 127.7 (2C; C-m’), 127.4 (2C; C-m), 126.7 (2C; C-o’), $123.4\left(\mathrm{C}-p^{\prime}\right), 114.3(\mathrm{C} \equiv \mathrm{N})$, $72.2(\mathrm{C}-4), 20.6\left(\mathrm{CH}_{2}\right), 18.3\left(\mathrm{Ar}-\mathrm{CH}_{3}\right), 12.2\left(\mathrm{CH}_{2} \mathrm{CH}_{3}\right) .{ }^{15} \mathrm{~N}$ NMR: Table 2. MS (EI, $\left.70 \mathrm{eV}\right): \mathrm{m} / z$ $(\%)=343\left(\mathrm{M}^{+}, 31\right), 207$ (100), 153 (20), $136(15), 104$ (5). Anal. Calcd for $\mathrm{C}_{21} \mathrm{H}_{21} \mathrm{~N}_{5}$ (343.18): C, 73.44; H, 6.16; N, 20.39. Found: C, 73.24; H, 6.00; N, 20.30.

(E)-5-Amino-1-((4'-chlorophenylimino)(phenyl)methyl)-3-ethyl-1H-pyrazole-4-carbonitrile (3h). Pale yellow crystals (EtOAc), yield: $0.27 \mathrm{~g}(72 \%)$; mp 215-216 ${ }^{\circ} \mathrm{C}$. IR $\left(\mathrm{KBr} ; v_{\max }, \mathrm{cm}^{-1}\right)$ : 3369-3273 $\left(\mathrm{NH}_{2}\right), 3060(\mathrm{CH}$ aromatic), $2980(\mathrm{CH}$ aliphatic), $2223(\mathrm{C} \equiv \mathrm{N}), 1647,1620(\mathrm{C}=\mathrm{N})$, $1556(\mathrm{C}=\mathrm{C}) .{ }^{1} \mathrm{H}$ NMR (DMSO-d $)$ ): $\delta_{H} 8.18$ (bs, 2H, NH ), 7.35-7.25 (m, 5H, H-o,m,p), 7.20-7.18 (d, $J 8.5 \mathrm{~Hz}, 2 \mathrm{H}$; H- $m^{\prime}$ ), 6.80-6.77 (d, J 8.5 Hz, 2H; H-o ), 2.45-2.40 (q, J 7.6 Hz, 2H; $\mathrm{CH}_{2}$ ), 1.091.05 (t, $\left.J 7.5 \mathrm{~Hz}, 3 \mathrm{H} ; \mathrm{CH}_{3}\right) .{ }^{13} \mathrm{C}$ NMR (DMSO- $\left.d_{6}\right): \delta_{C} 158.1,155.4,155.1,145.6,130.6,129.5$, 129.2 (2C), 128.6, 128.3 (2C), 127.6 (2C), 123.4 (2C), 114.3, 72.0, 20.5, 12.2. (EI, $70 \mathrm{eV}): \mathrm{m} / \mathrm{z}$ $(\%)=350\left(\mathrm{M}^{+}, 8\right), 349(30), 314$ (22), 238 (100), 162 (18), 77 (40). Anal. Calcd for $\mathrm{C}_{19} \mathrm{H}_{16} \mathrm{ClN}_{5}$ (349.11): C, 65.24; H, 4.61; N, 20.02. Found: C, 65.20; H, 4.55; N, 19.90.

(E)-5-Amino-3-ethyl-1-((4'-nitrophenylimino)(phenyl)methyl)-1H-pyrazole-4-carbonitrile (3i). Pale yellow crystals (EtOAc), yield: $0.26 \mathrm{~g}$ (71\%); mp 262-263 ${ }^{\circ} \mathrm{C}$; IR ( $\left.\mathrm{KBr} ; v_{\max }, \mathrm{cm}^{-1}\right)$ : 3335-3237 $\left(\mathrm{NH}_{2}\right), 3109(\mathrm{CH}$ aromatic), $2968(\mathrm{CH}$ aliphatic), $2216(\mathrm{C} \equiv \mathrm{N}), 1651,1600(\mathrm{C}=\mathrm{N})$, $1559(\mathrm{C}=\mathrm{C}) .{ }^{1} \mathrm{H}$ NMR (DMSO-d $): \delta_{H} 8.18\left(\mathrm{bs}, 2 \mathrm{H} ; \mathrm{NH}_{2}\right), 8.05(\mathrm{~d}, J 8.8 \mathrm{~Hz}, 2 \mathrm{H}$; (H-m'), $7.31(\mathrm{~m}$, $5 \mathrm{H}$; H-o,m,p), 7.03 (d, J 8.8 Hz, 2H; H-o'), 2.44 (q, J 7.6 Hz, 2H; $\mathrm{CH}_{2}$ ), 1.09 (t, J 7.6 Hz, 3H; $\mathrm{CH}_{3}$ ). ${ }^{13} \mathrm{C}$ NMR (DMSO- $\left.d_{6}\right): \delta_{C} 157.8$ (C- $\alpha$ ), 155.9 (C-3), 155.1 (C-5), 153.4 (C-p'), $143.2\left(\mathrm{C}-i^{\prime}\right)$, 130.4 (C-i), 129.8 (C-p), 129.3 (2C), 127.7 (2C; C-o,m), 124.2 (2C; C-m'), 122.6 (2C; C-o'), 114.2 $(\mathrm{C} \equiv \mathrm{N}), 72.1(\mathrm{C}-4), 20.6\left(\mathrm{CH}_{2}\right), 12.2\left(\mathrm{CH}_{3}\right) .{ }^{15} \mathrm{~N}$ NMR: Table 2. MS (EI, $\left.70 \mathrm{eV}\right): \mathrm{m} / z(\%)=360$ ( $\left.\mathrm{M}^{+}, 45\right), 225$ (20), 224 (100), 153 (78), 135 (55), 106 (16), 90 (13). Anal. Calcd for $\mathrm{C}_{19} \mathrm{H}_{16} \mathrm{~N}_{6} \mathrm{O}_{2}$ (360.13): C, 63.32; H, 4.48; N, 23.32. Found: C, 63.20; H, 4.40; N, 23.20.

(E)-5-Amino-1-((4'-bromophenylimino)(phenyl)methyl)-3-ethyl-1H-pyrazole-4-carbonitrile (3j). Pale yellow crystals (EtOAc), yield: $0.28 \mathrm{~g}$ (72\%); mp 200-201 ${ }^{\circ} \mathrm{C}$. IR ( $\left.\mathrm{KBr} ; v_{\max }, \mathrm{cm}^{-1}\right)$ : 3367-3272 $\left(\mathrm{NH}_{2}\right), 3050(\mathrm{CH}$ aromatic), $2990(\mathrm{CH}$ aliphatic), $2222(\mathrm{C} \equiv \mathrm{N}), 1647,1613(\mathrm{C}=\mathrm{N})$, $1556(\mathrm{C}=\mathrm{C}) .{ }^{1} \mathrm{H}$ NMR (DMSO-d $): \delta_{H} 8.1$ (bs, 2H; NH ), $7.30(\mathrm{~m}, 5 \mathrm{H} ; \mathrm{H}-o, m, p), 7.20$ (d, $J$ 8.3, 2H; H-m ), 6.79 (d, J 8.2, 2H; H-o'), 2.43 (q, J 7.5 Hz, 2H; $\left.\mathrm{CH}_{2}\right), 1.09$ (t, J 7.4 Hz, 3H; $\left.\mathrm{CH}_{3}\right) .{ }^{13} \mathrm{C}$ NMR (DMSO- $\left.d_{6}\right): \delta_{C} 157.9,155.1,146.1,145.6,131.2,130.6,129.5,129.3$ (2C), 128.3 (2C), 127.6 (2C), 123.4 (2C), 115.9, 114.3, 72.0, 20.6, 12.2 ppm. ${ }^{15} \mathrm{~N}$ NMR: Table 2. MS (EI, $\left.70 \mathrm{eV}\right)$ : $m / z(\%)=395(\mathrm{M}+2,35), 393\left(\mathrm{M}^{+}, 50\right), 349$ (21), 306 (32), 257 (87), 213 (46), 153 (100), 135 (67), 106 (19), 90 (12). Anal. Calcd for $\mathrm{C}_{19} \mathrm{H}_{16} \mathrm{BrN}_{5}$ (393.06): C, 57.88; H, 4.09; N, 17.76. Found: C, 57.70; H, 3.90; N, 17.60.

(E)-5-Amino-3-methyl-1-(phenyl(o-tolylimino)methyl)-1H-pyrazole-4-carbonitrile (3k). Pale yellow crystals $\left(\mathrm{CH}_{3} \mathrm{OH} / \mathrm{DCM}\right)$, yield: $0.25 \mathrm{~g}(80 \%)$; mp 213-214 ${ }^{\circ} \mathrm{C}$. IR $\left(\mathrm{KBr} ; v_{\max }, \mathrm{cm}^{-1}\right)$ : $3347-$ 
$3257\left(\mathrm{NH}_{2}\right), 3063(\mathrm{CH}$ aromatic), $2966(\mathrm{CH}$ aliphatic), $2219(\mathrm{C} \equiv \mathrm{N}), 1653,1621(\mathrm{C}=\mathrm{N}), 1560$ $(\mathrm{C}=\mathrm{C}) .{ }^{1} \mathrm{H}$ NMR (DMSO-d $)_{\text {) }} \delta_{H} 8.23$ (bs, 2H; NH $\mathrm{N}_{2}$ ), 7.28 (m, 3H; H-m,p), 7.23 (m, 2H; H-o), 7.09 (d, J 7.1 Hz, 1H; H-6'), 6.90 (ddd, J 7.5, 7.5, 1.1 Hz, 1H; H-4'), 6.84 (ddd, J 7.3, 7.3, 1.2 Hz, 1H; H-5'), 6.53 (dd, J 7.6, $0.9 \mathrm{~Hz}, 1 \mathrm{H}$; H-3'), 2.17 (s, 3H; Ar-CH 3$), 2.05$ (s, 3H; pyrazole-CH$).{ }^{13} \mathrm{C}$ NMR (DMSO- $\left.d_{6}\right): \delta_{C} 157.1(\mathrm{C}-\alpha), 154.9(\mathrm{C}-5), 150.3$ (C-3), 145.4 (C-2'), $131.1(\mathrm{C}-i), 129.8(\mathrm{C}-$ 6'), 129.4 (C-p), 128.7 (2C; C-o/m), 128.5 (C-1'), 127.6 (2C; C-m/o), 125.8 (C-4'), 123.7 (C-5'), 120.8 C-3'), $114.3(\mathrm{C} \equiv \mathrm{N}), 73.4(\mathrm{C}-4), 18.0\left(\mathrm{Ar}-\mathrm{CH}_{3}\right), 12.6\left(\right.$ pyrazole- $\left.\mathrm{CH}_{3}\right) .{ }^{15} \mathrm{~N}$ NMR: Table 2. MS (EI, $70 \mathrm{eV}): m / z(\%)=315\left(\mathrm{M}^{+}, 34\right), 193(100), 90$ (13). Anal. Calcd for $\mathrm{C}_{19} \mathrm{H}_{17} \mathrm{~N}_{5}(315.15)$ : C, 72.36; H, 5.43; N, 22.21. Found: C, 72.20; H, 5.30; N, 22.10.

(E)-5-Amino-1-((2',6'-dimethylphenyl)imino)(phenyl)methyl)-3-methyl-1H-pyrazole-4carbonitrile (3l). Pale yellow crystals $\left(\mathrm{CH}_{3} \mathrm{OH} / \mathrm{DCM}\right)$, yield: $0.26 \mathrm{~g}(81 \%)$; mp 275-276 ${ }^{\circ} \mathrm{C}$. IR $\left(\mathrm{KBr} ; v_{\max }, \mathrm{cm}^{-1}\right): 3362-3277\left(\mathrm{NH}_{2}\right), 3060(\mathrm{CH}$ aromatic), $2946(\mathrm{CH}$ aliphatic $), 2213(\mathrm{C} \equiv \mathrm{N}), 1652$, $1621(\mathrm{C}=\mathrm{N}), 1558(\mathrm{C}=\mathrm{C}) .{ }^{1} \mathrm{H}$ NMR (DMSO- $\left.d_{6}\right): \delta_{H} 8.16\left(\mathrm{bs}, 2 \mathrm{H} ; \mathrm{NH}_{2}\right), 7.33(\mathrm{tt}, J 7.3,2.2 \mathrm{~Hz}, 1 \mathrm{H}$; H-p), 7.26 (dd, J 7.7, 7.2 Hz, 2H; H-m), 7.18 (d, J 7.2 Hz, 2H; H-o), 6.90 (d, J 7.5 Hz, 2H; H-m'), $6.79\left(\mathrm{t}, J 7.5 \mathrm{~Hz}, 1 \mathrm{H} ; \mathrm{H}-p\right.$ '), 2.07 (s, 3H; pyrazole- $\left.\mathrm{CH}_{3}\right), 2.04$ (s, 6H; $\left.\mathrm{Ar}-\mathrm{CH}_{3}\right) .{ }^{13} \mathrm{C}$ NMR (DMSO$\left.d_{6}\right): \delta_{C} 156.1(\mathrm{C}-\alpha), 155.0(\mathrm{C}-5), 150.2(\mathrm{C}-3), 144.3(\mathrm{C}-i$ '), $131.4(\mathrm{C}-i), 129.2(\mathrm{C}-p), 128.2(2 \mathrm{C}$; C$o), 127.7$ (2C; C-m’), 127.5 (2C; C-m), 126.6 (2C; C-o'), 123.4 (C-p’), $114.3(\mathrm{C} \equiv \mathrm{N}), 73.5(\mathrm{C}-4)$, $18.3\left(\mathrm{Ar}_{-} \mathrm{CH}_{3}\right), 12.6$ (pyrazole- $\left.\mathrm{CH}_{3}\right) .{ }^{15} \mathrm{~N}$ NMR: Table 2. MS (EI, $\left.70 \mathrm{eV}\right) \mathrm{m} / \mathrm{z}(\%)=329\left(\mathrm{M}^{+}, 37\right)$, 207 (100), 106 (20). Anal. Calcd for $\mathrm{C}_{20} \mathrm{H}_{19} \mathrm{~N}_{5}$ (329.16): C, 72.93; H, 5.81; N, 21.26. Found: C, $72.80 ; \mathrm{H}, 5.70 ; \mathrm{N}, 21.40$.

(E)-5-Amino-1-(((4'-chlorophenyl)imino)(phenyl)methyl)-3-methyl-1H-pyrazole-4-carbonitrile (3m). Pale yellow crystals $\left(\mathrm{CH}_{3} \mathrm{OH} / \mathrm{DCM}\right)$, yield: $0.25 \mathrm{~g}(76 \%)$; mp $257-258{ }^{0} \mathrm{C}$. IR $(\mathrm{KBr}$; $\left.v_{\max }, \mathrm{cm}^{-1}\right): 3357-3257\left(\mathrm{NH}_{2}\right), 3100(\mathrm{CH}$ aromatic), $2930(\mathrm{CH}$ aliphatic $), 2223(\mathrm{C} \equiv \mathrm{N}), 1642,1614$ $(\mathrm{C}=\mathrm{N}), 1559(\mathrm{C}=\mathrm{C}) .{ }^{1} \mathrm{H}-\mathrm{NMR}\left(\mathrm{DMSO}-d_{6}\right): \delta_{H} 8.19\left(\mathrm{bs}, 2 \mathrm{H} ; \mathrm{NH}_{2}\right), 7.31(\mathrm{~m}, 3 \mathrm{H} ; \mathrm{H}-m, p), 7.26$ (dd, $J$ 7.7, 1.7 Hz, 2H; H-o), 7.20 (d, J 8.6 Hz.2H; H-m'), 6.79 (d, J 8.6 Hz, 2H; H-o'), 2.05 (s, 3H; pyrazole- $\mathrm{CH}_{3}$ ). ${ }^{13} \mathrm{C}-\mathrm{NMR}$ (DMSO- $\left.d_{6}\right): \delta_{\mathrm{C}} 158.0(\mathrm{C}-\alpha), 154.8(\mathrm{C}-5), 150.5(\mathrm{C}-3), 145.6\left(\mathrm{C}-i^{\prime}\right)$, 130.7 (C-i), 129.5 (C-p), 129.2 (2C; C-o), 128.3 (2C; C-m'), 127.74 (C-i), 127.68 (2C; C-m), 123.5 (2C; C-o'), $114.3(\mathrm{C} \equiv \mathrm{N}), 73.3(\mathrm{C}-4), 12.6\left(\mathrm{CH}_{3}\right) .{ }^{15} \mathrm{~N}$ NMR: Table 2. MS (EI, $\left.70 \mathrm{eV}\right): \mathrm{m} / \mathrm{z}(\%)=$ $336\left(\mathrm{M}^{+}, 8\right), 335\left(\mathrm{M}^{+}, 30\right), 301$ (22), 300 (18), 225 (100), 150 (24), 124 (22), 110 (24). Anal. Calcd for $\mathrm{C}_{18} \mathrm{H}_{14} \mathrm{ClN}_{5}$ (335.09): C, 64.38; H, 4.20; N, 20.86. Found: C, 64.20; H, 4.00; N, 20.70.

(E)-5-Amino-3-methyl-1-((4'-nitrophenyl)imino)(phenyl)methyl)-1H-pyrazole-4-carbonitrile (3n). Pale yellow crystals $\left(\mathrm{CH}_{3} \mathrm{OH} / \mathrm{DCM}\right)$, yield: $0.26 \mathrm{~g}(76 \%)$; mp 279-280 ${ }^{\circ} \mathrm{C}$. $\mathrm{IR}(\mathrm{KBr}$; $\left.v_{\max }, \mathrm{cm}^{-1}\right)$ : 3394-3294 $\left(\mathrm{NH}_{2}\right), 3067$ (CH aromatic), $2924(\mathrm{CH}$ aliphatic), $2212(\mathrm{C} \equiv \mathrm{N}), 1659,1602$ $(\mathrm{C}=\mathrm{N}), 1557(\mathrm{C}=\mathrm{C}) .{ }^{1} \mathrm{H}$ NMR (DMSO-d6): $\delta_{H} 8.18$ (bs, 2H; $\left.\mathrm{NH}_{2}\right), 8.05$ (d, $\left.J 8.8 \mathrm{~Hz}, 2 \mathrm{H} ; \mathrm{H}-m^{\prime}\right)$, $7.31(\mathrm{~m}, 5 \mathrm{H} ; \mathrm{H}-o, m, p), 7.03$ (d, J 8.9 Hz, 2H; H-o '), 2.07 (s, 3H; $\left.\mathrm{CH}_{3}\right) .{ }^{13} \mathrm{C}$ NMR (DMSO-d $): \delta_{\mathrm{C}}$ 157.8 (C- $\alpha$ ), 154.9 (C-5), 153.3 (C-p’), 151.0 (C-3), 143.2 (C-i’), 130.5 (C-i), 129.8 (C-p), 129.2 (2C), $127.7(2 \mathrm{C} ; \mathrm{C}-m, o), 124.2\left(2 \mathrm{C} ; \mathrm{C}-m^{\prime}\right), 122.6\left(2 \mathrm{C} ; \mathrm{C}-o^{\prime}\right), 114.2(\mathrm{C} \equiv \mathrm{N}), 73.4(\mathrm{C}-4), 12.6\left(\mathrm{CH}_{3}\right)$. ${ }^{15} \mathrm{~N}$ NMR: Table 2. MS (EI, $\left.70 \mathrm{eV}\right): m / z(\%)=346\left(61, \mathrm{M}^{+}\right), 306(32), 224$ (92), $153(100), 136$ (65), 106 (18). Anal. Calcd for $\mathrm{C}_{18} \mathrm{H}_{14} \mathrm{~N}_{6} \mathrm{O}_{2}$ (346.12): C, 62.42; H, 4.07; N, 24.27. Found: C, 62.30; H, 4.20; N, 24.10. 


\section{(E)-5-Amino-1-(((4'-bromophenyl)imino)(phenyl)methyl)-3-methyl-1H-pyrazole-4-}

carbonitrile (3o). Pale yellow crystals $\left(\mathrm{CH}_{3} \mathrm{OH} / \mathrm{DCM}\right)$, yield: $0.29 \mathrm{~g}(75 \%)$; mp 250-251 ${ }^{\circ} \mathrm{C}$. IR $\left(\mathrm{KBr} ; v_{\max }, \mathrm{cm}^{-1}\right): 3370-3272\left(\mathrm{NH}_{2}\right), 3100(\mathrm{CH}$ aromatic), $2925(\mathrm{CH}$ aliphatic $), 2219(\mathrm{C} \equiv \mathrm{N}), 1642$, $1614(\mathrm{C}=\mathrm{N}), 1558(\mathrm{C}=\mathrm{C}) .{ }^{1} \mathrm{H}$ NMR (DMSO-d $)$ ): $\delta_{H} 8.19$ (bs, 2H; $\left.\mathrm{NH}_{2}\right), 7.32\left(\mathrm{~m}, 5 \mathrm{H} ; \mathrm{H}-m, p, m^{\prime}\right)$, $7.26\left(\mathrm{dd}, J\right.$ 7.8, $1.7 \mathrm{~Hz}, 2 \mathrm{H} ; \mathrm{H}-o$ ), 6.79 (d, $J 8.6 \mathrm{~Hz}, 2 \mathrm{H} ; \mathrm{H}-o$ '), 2.05 (s, 3H; $\mathrm{CH}_{3}$ ). ${ }^{13} \mathrm{C}$ NMR (DMSO- $\left.d_{6}\right): \delta_{C} 158.0(\mathrm{C}-\alpha), 154.8(\mathrm{C}-5), 150.5(\mathrm{C}-3), 146.0(\mathrm{C}-i$ '), 131.2 (2C; C-m), $130.7(\mathrm{C}-i)$, 129.5 (C-p), 129.2 (2C; C-o), 127.7 (2C; C-m'), 123.9 (C-o'), $115.9\left(\mathrm{C}^{\prime} p^{\prime}\right), 114.3(\mathrm{C} \equiv \mathrm{N}), 73.3(\mathrm{C}-$ 4), $12.6\left(\mathrm{CH}_{3}\right) .{ }^{15} \mathrm{~N}$ NMR: Table 2. MS (EI, $\left.70 \mathrm{eV}\right): m / z(\%)=381(\mathrm{M}+2,34), 379\left(\mathrm{M}^{+}, 36\right), 259$ (100), 106 (14). 88 (9). Anal. Calcd for $\mathrm{C}_{18} \mathrm{H}_{14} \mathrm{BrN}_{5}$ (379.04): C, 56.86; H, 3.71; N, 18.42. Found: C, 56.70; H, 3.70; N, 18.30 .

\section{Conclusions}

$N$-Arylbenzamidrazones reacted with 2-(ethoxymethylene)malononitriles to furnish the corresponding pyrazoles. The regiochemistry of the products requires that the process begin with attack by the remote nitrogen of the benzamidrazone, on the methylenemalononitrile in conjugate fashion.

\section{Acknowledgements}

The NMR spectrometer at Florida Institute of Technology was purchased with the assistance of the National Science Foundation (CHE 03-42251).

\section{References}

1. Aly, A. A.; Gomaa, A.-M. M.; Nour-El-Din, A. M.; Fahmy, M. S. Arkivoc 2007, (xvi), 41-50. http://dx.doi.org/10.3998/ark.5550190.0008.g04

2. Aly, A. A.; Nour El-Din, A. M.; Gomaa, M. A.-M.; Fahmi, M. S. Z. Naturforsch. 2008, 63B, 223-228.

3. Nour El-Din, A. M.; Aly, A. A. J. Chem. Res. 2007, 665-667. https://doi.org/10.3184/030823407X268340

4. Aly, A. A.; Hassan, A. A.; Bräse, S.; Gomaa, M. A.-M.; Nemr, F. M. J. Heterocycl. Chem. 2016, in press.

http://doi.org /10.1002/jhet.2607.

5. Aly, A. A.; Hassan, A. A.; Gomaa, M. A.-M.; Bräse, S.; Nemr, F. M. J. Heterocycl. Chem. 2016 in press.

http://doi.org/10.1002/jhet.2643 
6. Aly, A. A.; Ramadan, M.; Abd Al-Aziz, M.; Fathy, H. M.; Bräse, S.; Brown, A. B.; Nieger, M. J. Chem. Res. 2016, 40, 637-639. https://doi.org/10.3184/174751916X14743924874916

7. Elguero, J. In Comprehensive Heterocyclic Chemistry II, Shinkai, I., Ed.; Elsevier: Oxford, 1996; Vol. 3, pp 1-75, 817-932.

8. Knorr, L. Justus Liebigs Ann. Chem. 1887, 238, 137-219. https://doi.org/10.1002/jlac.18872380107

9. Baynes, R. E.; Dedonder, K.; Kissell, L.; Mzyk, D.; Marmulak, T.; Smith, G.; Tell, L.; Gehring, R.; Davis, J.; Riviere, J. E. Food Chem. Toxicol. 2016, 88, 112-122. https://doi.org/10.1016/j.fct.2015.12.020

10. Kendre, B. V.; Landge, M. G.; Bhusare, S. R. Arab. J. Chem. 2016 in press. http://doi.org/10.1016/j.arabjc.2015.01.007

11. Tewari, A. K.; Singh V. P.; Yadav, P.; Gupta, G.; Singh, A.; Goel, R. K. Bioorg. Chem. 2014, $56,8-15$. https://doi.org/10.1016/j.bioorg.2014.05.004

12. Ragavan, R. V.; Vijayakumar, V.; Kumari, N. S. Eur. J. Med. Chem. 2010, 45, 1173-1180. https://doi.org/10.1016/j.ejmech.2009.12.042

13. Christodoulou, M. S.; Liekens, S.; Kasiotis, K. M.; Haroutounian, S. A. Bioorg. Med. Chem. 2010, 18, 4338-4350. https://doi.org/10.1016/j.bmc.2010.04.076

14. Xia, Y.; Dong Z. W.; Zhao, B. X.; Ge, X.; Meng, N.; Shin. D. S. Bioorg. Med. Chem. 2007, 15, 6893-6899. https://doi.org/10.1016/j.bmc.2007.08.021

15. Lv, P. C.; Li, H. Q.; Sun, J.; Zhou, Y.; Zhu, H. L. Bioorg. Med. Chem. 2010, 18, 4606-4614. https://doi.org/10.1016/j.bmc.2010.05.034

16. Abdel-Aziz, M.; Abuo-Rahma, G.-D.; Hassan, A. A. Eur. J. Med. Chem. 2009, 44, 3480-3470. https://doi.org/10.1016/j.ejmech.2009.01.032

17. Kotsuki, H.; Wakao, M.; Hayakawa, H.; Shimanouchi, T.; Shiro, M. J. Org. Chem. 1996, 61, 8915-8920.

https://doi.org/10.1021/jo961388c

18. Togni, A.; Burckhardt, U.; Gramlich, V.; Pregosin, P. S.; Salzmann, R. J. Am. Chem. Soc. 1996, 118 1031-1037. https://doi.org/10.1021/ja953031g

19. Yu, W. S.; Cheng, C. C.; Cheng, Y. M.; Wu, P. C.; Song, Y. H.; Chi, Y.; Chou, P. T. J. Am. Chem. Soc. 2003, 125, 10800-10801. https://doi.org/10.1021/ja953031g

20. Reviriego, F.; Rodríguez-Franco, M. I.; Navarro, P.; García-España, E.; Liu-González, M.; Verdejo, B.; Domènech, A. J. Am. Chem. Soc. 2006, 128, 16458-16459.

https://doi.org/10.1021/ja064657h 
21. Chin, J.; Oh, J.; Jon, S. Y.; Park, S. H.; Walsdorff, C.; Stranix, B.; Ghoussoub, A.; Lee, S. J.; Chung, H. J.; Park, S. M.; Kim, K. J. Am. Chem. Soc. 2002, 124, 53-74.

21. Trofimenko, S. Chem. Rev. 1993, 93, 943-980.

https://doi.org/10.1021/cr00019a006

22. Han, S. J.; Kim, H. T.; Joo, J. M. J. Org. Chem. 2016, 81, 689-698.

https://doi.org/10.1021/acs.joc.5b02398

23. Doyle, K. M.; Kurzer F. Synthesis 1974, 383-384.

24. Kleinpeter, E.; Koch, A.; Heydenreich, M.; Chatterjee, S. K.; Rudorf, W.-D. J. Mol. Struct. 1995, 356, 25-33, and references therein.

https://doi.org/10.1016/0022-2860(95)08919-M

25. Berger, S.; Braun, S. 200 and More NMR Experiments; Wiley-VCH: Weinheim, 2004; pp 415421, 538-545, 673-677.

26. Berger, S.; Braun, S.; Kalinowski, H.-O. NMR Spectroscopy of the Non-Metallic Elements, John Wiley \& Sons: Chichester, 1997; pp 242-262.

27. Furrer, J. Concepts Magn. Reson. (Part A) 2015, 43A, 177-206.

28. Oszczapowicz, J.; Wawer, I.; Dargatz, M.; Kleinpeter, E. J. Chem. Soc., Perkin Trans. 2 1995, 1127-1131. https://doi.org/10.1016/0022-2860(95)08919-M

29. Bovey, F. A. Nuclear Magnetic Resonance Spectroscopy, $2^{\text {nd }}$ Ed.; Academic: San Diego, 1988; p 461. 